$\xi=-1$

\title{
Flow and Heat Dissipation Analysis in Transformer Substation with Minimal Ventilation using CFD
}

\author{
Farid Akmal Saipul Bahri ${ }^{1}$, Hasril Hasini ${ }^{2 *}$ \\ ${ }^{I}$ Department of Mechanical Engineering, Universiti Tenaga Nasional, Jalan Kajang-UNITEN, \\ 43000 Kajang, Selangor, MALAYSIA \\ ${ }^{2}$ Fluid Dynamics and Disaster Risk Management Research Group, Institute of Sustainability Engineering, \\ Universiti Tenaga Nasional, 43000 Kajang, Selangor, MALAYSIA \\ *Corresponding Author E mail: hasril@uniten.edu.my
}

\begin{abstract}
This paper aims to investigate the flow and heat dissipation of a transformer located in substation building with minimal ventilation system. The study is carried out using CFD, which model the flow and conjugate heat transfer in step-up transformer and its surrounding air. Transformer model is generated by considering the extended fins at both side which enhance heat dissipation. Three different substation ventilation designs are proposed in this work. The effect of air outlet positions with fixed inlets are investigated. The primary objective is to investigate transformer surface and surrounding temperatures for different ventilation systems. The result indicated that minimum natural ventilation of a transformer substation is achievable to avoid transformer overheating. This must be achieved by carefully selecting suitable air inlets and outlets positions in a transformer substation.
\end{abstract}

Keywords: CFD, Transformer substation, Natural ventilation

\section{Introduction}

A transformer is a device which is commonly used in various engineering applications such as in electricity generation, transmission and distribution. It uses the principle of electromagnetic induction to convert an alternating current $(\mathrm{A} / \mathrm{C})$ with certain voltage to similar current of different voltage. This device operates by transmitting electrical signals without changing the frequency to the other circuit with minimal power losses due to heat generation from the conversion alternating current. The transformer is usually located in a building to protect it from sensitive contaminant such as corrosive gases, sand, dust, rain, etc. that will reduce its operating life time.

There are many types of transformer and among the most widely used one are oil-cooled and dry transformer. For most indoor-type transformer, dry-type transformer is preferable to avoid the risk of fire in the building. It is known that the oil-type transformer is prone to fire hazard as oil used as the cooling medium is flammable. The dry-type transformer, on the other hand uses air as the cooling medium. Usually the air is forced through fan or airconditioning devices installed inside the building. These devices however, needs to be operated 24 hours a day, depending on the load imposed on the transformer. The gap or openings that allows air to enter and leaves the building enables unwanted particle or dust to enter as well. Even though the gap or opening is supported by filters, the accumulation of dust or foreign particles on transformer surface posed hazards as it forms thin layer on transformer surface, reducing convection coefficient. The transformer will risk getting unnecessarily hot with the existing cooling conditions.

The problem was first reported in one of the substation in East Coast of Malaysia where it was discovered that failure to transformer occurred due to heavy dust and particle from the surround- ing environment. The substation was originally designed to operate under moderate surrounding condition with a single exhaust fan installed to force the air into the building for transformer cooling. However, due to an increase in mining activity in the surrounding vicinity, severe dust and particle has plague the area and this affect the operation of transformer. Frequent tripping of transformer was reported due to overheating from severe dust accumulation.

One of the possible methods to prevent dust and foreign particle to enter the substation is by minimizing the opening of air gaps in the building. However, careful consideration must be made to ensure that the closing of existing air channel and intakes does not compromise the ability of the new ventilation system to dissipate heat from the transformer. Thus this work aims to investigate the potential of having minimal ventilation in a transformer substation, sufficient to ensure safe operation of the transformer. In the open literature, limited number of work were found on the study of optimum ventilation of transformer building. A few works were found on the investigation of the efficiency of indoor substation by simulation [1-2]. It was concluded that height elevation between air outlet and inlet will improve the indoor temperature and velocity fields [2]. For a typical transformer heat map, the hottest spot was usually located at the top surface, followed by the sides of transformer [3]. The closest work on the optimization of air inlet and outlet openings was found in Ref. [4]. This work used numerical simulation to investigate the flow and heat transfer in the main transformer room of an indoor substation with three different outlet configurations.

In this paper, commercial CFD code is used to predict the velocity field and temperature field in transformer substation. The minimal ventilation was used in this analysis for three different configurations where location of air inlets is fixed while, the outlets varies. 


\section{Description of Simulation Model}

\subsection{Model Description and Simplification}

The transformer substation is modelled based on typical outdoor substation used in Malaysia. Typically, the substation consists of a few inlets and outlet openings. The substation was designed as a rectangular building with dimensions of $5.969 \mathrm{~m}$ (length), $5.969 \mathrm{~m}$ (width) and $4.166 \mathrm{~m}$ (height) with total volume is $148.42 \mathrm{~m}^{3}$. This assumption was made based on a typical sizing of a transformer substation. For consistency, the size of the inlet and outlet are made similar and the dimension is $1.219 \mathrm{~m} \times 0.584 \mathrm{~m}$ with total area $0.712 \mathrm{~m}^{2}$. The transformer was designed as a cuboid with 48 fins at both side. The dimension of the transformer without fin was $2.14 \mathrm{~m}$ long $\times 2.14 \mathrm{~m}$ wide $\times 1.27 \mathrm{~m}$ height and fin thickness is the $0.05 \mathrm{~m}$ and $0.4 \mathrm{~m}$ length. The fixed heat flux of $5,000 \mathrm{~W} / \mathrm{m}^{2}$ was set inside of the transformer based on the average transformer load variation throughout the day. Also, this number is chosen by assuming that heat is evenly distributed in all direction from the transformer core. The component of transformer model is simplified as cuboid with $1.5 \mathrm{~m}$ length $\times 1 \mathrm{~m}$ wide. The simplification is made close enough to actual geometry of transformer under investigation. For the purpose of analysis, a typical transformer substation model is shown in Fig. 1. The building is confined by 4 walls termed Front wall, Rear wall, Left wall and Right wall. The front wall is the wall that has air inlet openings, in parallel with the $x-z$ plane. The rear wall is located at the opposite but in parallel plane with the front wall. The left wall is the wall which is in parallel with the $y-z$ plane. While the rear wall is a wall located opposite the left wall, on the same $y-z$ plane.

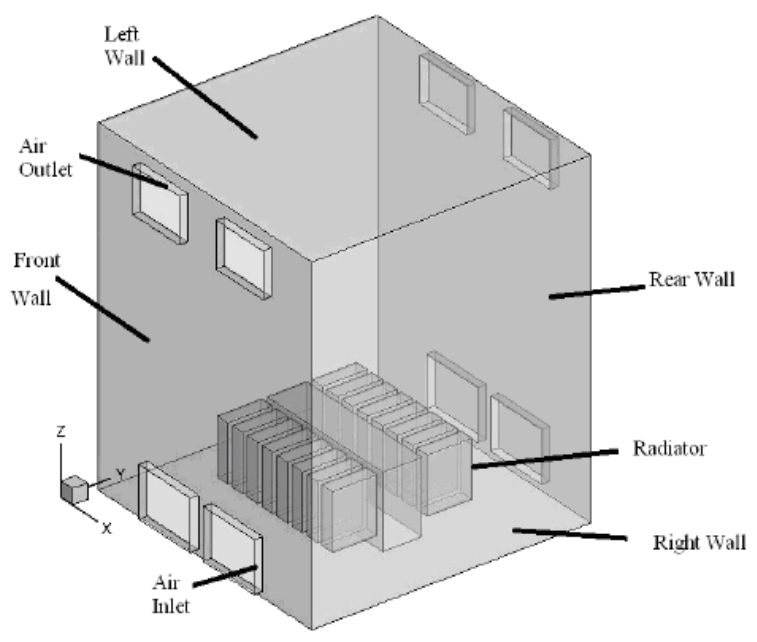

Fig 1: Schematics illustration of a typical transformer substation

\subsection{Ventilation Mode}

In this work, three outlets configurations with fixed inlets were tested. For simplicity, the two inlets were made fixed. Fig. 2 shows three substations with different positions of the air outlets, named Case A, Case B and Case C. In Case A, the air outlets are positioned on the rear wall of substation and located opposite with the air inlets. In Case B, the air outlets were located at the left wall and in Case C, the outlets are located at left and right walls each. The air inlets for three transformer substation were maintained at the same position while the transformer was maintained at the middle of the substation.

\subsection{Governing Equations}

The computational fluid dynamics simulation of flow in transformer substation employ standard Navier-Stokes equations describing continuity, momentum and energy equations [5]. Incompressible, three dimensional turbulence flow is assumed. The flow inside transformer substation involves single-phase fluid in gas phase. The finite volume method is adopted for the solution. In addition, the utilisation of second order upwind scheme is applied to continuity, momentum, turbulent kinetic energy, turbulent dissipation rate and energy equation. Standard k- $\varepsilon$ model and pressure-velocity coupling was found fit to be the implemented for the solution. The buoyant effect is taken into account due to significant temperature difference that is anticipated when air flows pass the transformer surface.

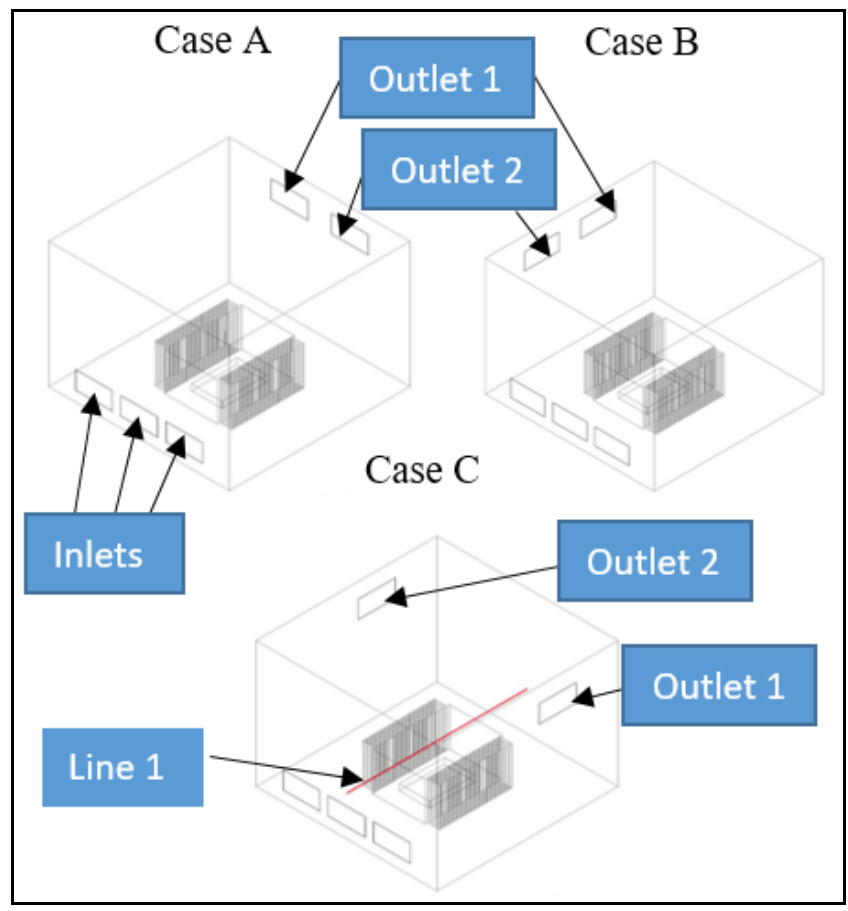

Fig. 2: Inlet and outlet arrangement designs for ventilation transforme substation.

\section{Continuity Equation}

According to Reynolds assumptions, the flow at any instant at each point in the flow field obeys the basic equation for continuum flow:

$\frac{\partial \rho}{\partial t}+\frac{\partial \rho u}{\partial x}+\frac{\partial \rho v}{\partial y}+\frac{\partial \rho w}{\partial z}=0$

Where the second, third, and fourth terms are the divergence of mass flow density and can be written as vector symbols:

$$
\frac{\partial \rho}{\partial t}+\nabla(\rho \vec{u})=0
$$

The density of incompressible fluid is a constant, and the continuity equation can be casted into the following forms:

$$
\nabla(\rho \vec{u})=0
$$

Where $\nabla$ is a differential operator, and $\vec{u}$ is a velocity vector.

\section{Differential Energy Equation}

For an infinitesimal control volume in a flow field, the differential energy equation can be obtained using the continuity equation according to the first law of thermodynamics:

$\rho c_{v}\left(\frac{\partial T}{\partial \tau}\right)+\nabla(a \nabla T+Q)$ 
Where $Q$ is the heat generated per unit volume, $T$ is temperature, $C_{v}$ is constant volume heat capacity, $\tau$ is a time constant, $\rho$ is density, $\alpha$ is the thermal diffusion coefficient, and $\partial T / \partial \tau$ is the change of energy with respect time.

\section{Differential Momentum Equation}

For an infinitesimal control volume in a flow field, the differential momentum equation (N-S equation) can be obtained using the continuity equation according to the momentum conservation law:

$\rho\left(\frac{\partial V}{\partial \tau}\right)=F-\nabla P+\mu \nabla^{2} V$

Where $\mu$ is the dynamic viscosity coefficient, $P$ is pressure, $\rho(\partial V / \partial \tau)$ is inertia force, $F$ is the buoyancy force caused by uneven density, $\nabla P$ is the total pressure gradient, and $\mu \nabla^{2} V$ is viscous force.

\subsection{Boundary Conditions}

The air inlets were set as weak flow with inlet velocity of $0.01 \mathrm{~m} / \mathrm{s}$ each while the outlets are set as the pressure outlet. This assumption is based on actual assessment and measurement on-site. The assumption is also made to emulate the worst case scenario where lack of air could cause overheating of transformer. The temperature of air inlet is assumed at $300 \mathrm{~K}$. Heat source is assumed to originate from the transformer via the bottom surface. The heat flux is set at $5,000 \mathrm{~W} / \mathrm{m}^{2}$.based on the established heat load published by the manufacturer. No-slip condition is assumed for all walls. However, to ensure proper prediction of boundary layer at wall, wall function is applied with refined mesh. The interface boundary condition was created between transformer and the room surfaces to allow conjugate heat transfer calculation for conduction of heat through solid and coupled with convection heat transfer in fluid. The overall calculation for flow and conjugate hear transfer is carried out in steady state condition.

\subsection{Grid Dependency Study}

Prior to the extensive simulation, grid dependence test is conducted for Case A to ensure grid independent solution. 3 different mesh size were tested and the summary of grid sizes is shown in Table 1. For all cases, the maximum temperature at transformer surface is recorded. Similarly, quantitative observation of flow and temperature contours were compared. It was notice that the maximum temperature predicted by coarse, medium and fine meshes are $355.0 \mathrm{~K}, 345.1 \mathrm{~K}$ and $345.1 \mathrm{~K}$ respectively. It was also observed that there was an appreciable difference in temperature and velocity contours for coarse and medium mesh. However, similar contour was observed between the medium and fine meshes. Thus it can be concluded that medium mesh scheme is sufficient to accurately predict the heat and flow properties in this work. Thus the medium mesh scheme is adopted for the remaining simulations. The same number of nodes (approximately 1.1 million) are also applies to Case B and Case C.

Table 1: Grid dependency test

\begin{tabular}{|l|l|l|l|}
\hline Case & Coarse & Medium & Fine \\
\hline Node & 204,936 & 491,745 & $\begin{array}{l}1,094,50 \\
8\end{array}$ \\
\hline Element & $\begin{array}{l}1,076,85 \\
7\end{array}$ & $\begin{array}{l}2,630,82 \\
5\end{array}$ & $\begin{array}{l}5,413,07 \\
9\end{array}$ \\
\hline $\begin{array}{l}\text { Max T at transformer surface } \\
\text { (K) }\end{array}$ & 355.0 & 345.1 & 345.1 \\
\hline
\end{tabular}

\section{Result, Analysis and Discussion}

To compare the effectiveness of ventilation of the transformer substation at various outlet locations, a few planes of interest were created to best represent the critical flow and heat contours. This includes the temperature distribution at transformer top surface for hot-spot analysis [6]. The temperature and velocity distributions at $y-z$ plane $(x=2.98 \mathrm{~m})$ is create to represent the centre of substation. To analyse the ventilation effect of various designs, the streamline in each substation and outlets temperatures are used for comparison. Heat released from transformer is convected as air flow passes over its surface. Heat will be brought out to outside ambient environment via outlets located at different locations. The summary of predictions of air temperature at different outlets are shown in Table 2. Generally, the outlet temperature shows an increase in air temperature by $8-10 \mathrm{~K}$ from the inlets.

Table 2: Prediction of air outlet temperature

\begin{tabular}{|l|l|l|l|}
\hline Case & A & B & C \\
\hline Inlet air T(K) & 300.0 & 300.0 & 300.0 \\
\hline Outlet 1 air T (K) & 310.0 & 310.3 & 309.4 \\
\hline Outlet 2 air T (K) & 309.9 & 308.9 & 309.4 \\
\hline
\end{tabular}

\subsection{Velocity Distribution}

Fig. 3 shows the velocity vectors at mid plane for all cases simulated. Note that the air enters the building from left, hit the transformer and dispersed to the respective outlets due to pressure difference. A few regions of re-circulation can be seen and it can be said that the flow is fully turbulent, thus it is anticipated that heat transfer is optimum despite the weak inlet flow. Comparing the vector at transformer top surface, Case A shows very weak flow vector compared to Case B and C. This could be attributed by longer distance from the top surface to outlet duct for Case A compared to Case B and C. The flow is expected to dispersed immediately for Case A, leaving insufficient time to absorbed more heat.

The velocity contours at $y-z$ plane of transformer substation for all cases is shown in Fig. 4. For all cases, the air velocity at the inlet is $0.01 \mathrm{~m} / \mathrm{s}$. This results in outlet velocity of 0.01784 and 0.01794 $\mathrm{m} / \mathrm{s}$ at Outlet 1 and 2 respectively for Case A. Slightly higher velocity at the outlet is expected due to the change in density which is caused when the airflow picks up heat from the transformer surface. It is interesting to note that the speed of the air that enters the substation reduces abruptly as it hits the transformer surface and dispersed in all directions towards the outlets. However, the speed is virtually zero and this will give adverse effect to the cooling of transformer. In Case B, the outlet velocity is predicted to be $0.01853 \mathrm{~m} / \mathrm{s}$. Two large recirculation regions were formed, located upstream and downstream of transformer (Refer to Fig. 3 for comparison). In Case $\mathrm{C}$, the maximum velocity predicted at both outlets are $0.01845 \mathrm{~m} / \mathrm{s}$. It is noted that the magnitude does not differ much as compared to Case A and C. The air disperses as it hits the transformer and recirculation occur slightly downstream of the transformer. The velocity at the top of transformer is found to be very low. This can be viewed through the middle plan created at mid plane.

The velocity contour for all cases at plane $x-y$ is illustrated in Fig. 5 . This plane corresponds to constant building height, $z=2.083 \mathrm{~m}$. The flow direction is also labelled for clarity. The flow can be seen deflected after hitting the transformer surface and this is given by regions of higher velocity right after air enters the inlet. Case B shows the highest air flow in the vicinity of upstream transformer edge, while Case $\mathrm{A}$ and Case $\mathrm{C}$ show similar contours possibly due to the symmetricity effect for these cases. 


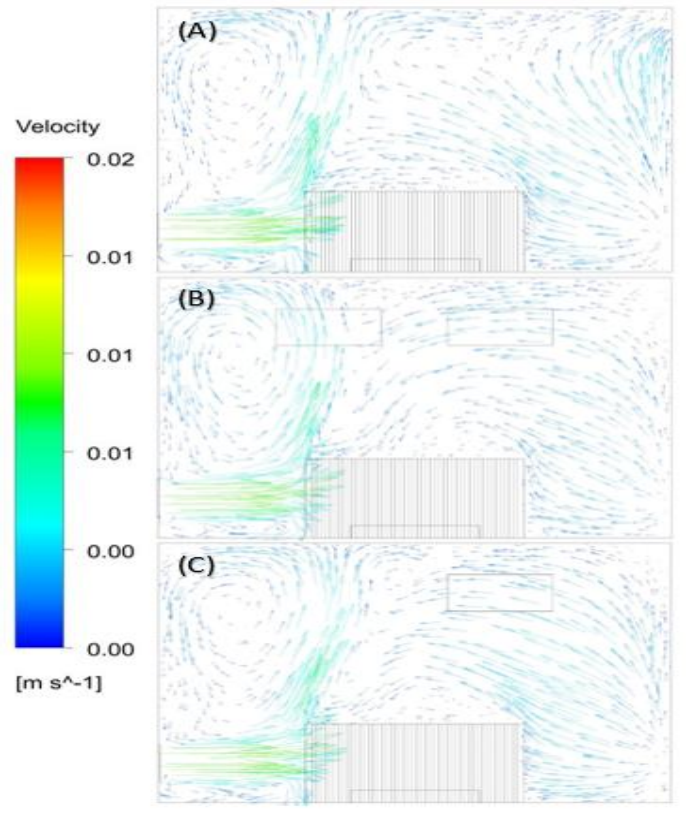

Fig 3: Velocity vector at plane $y-z(x=2.83 \mathrm{~m})$

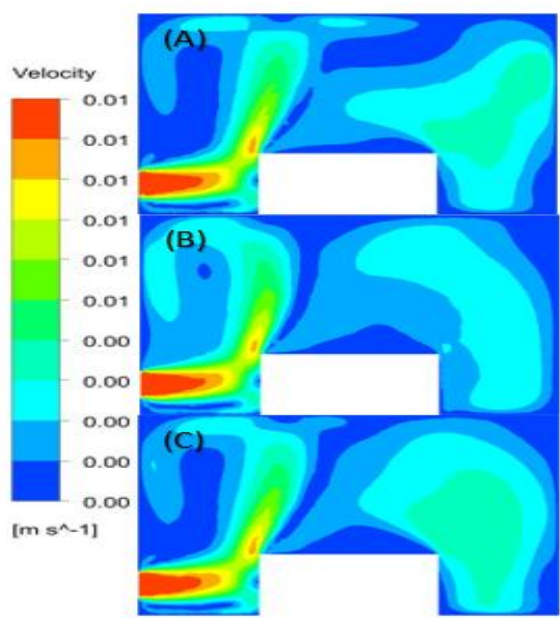

Fig 4: Velocity contour at $y-z$ plane $(x=2.98 \mathrm{~m})$

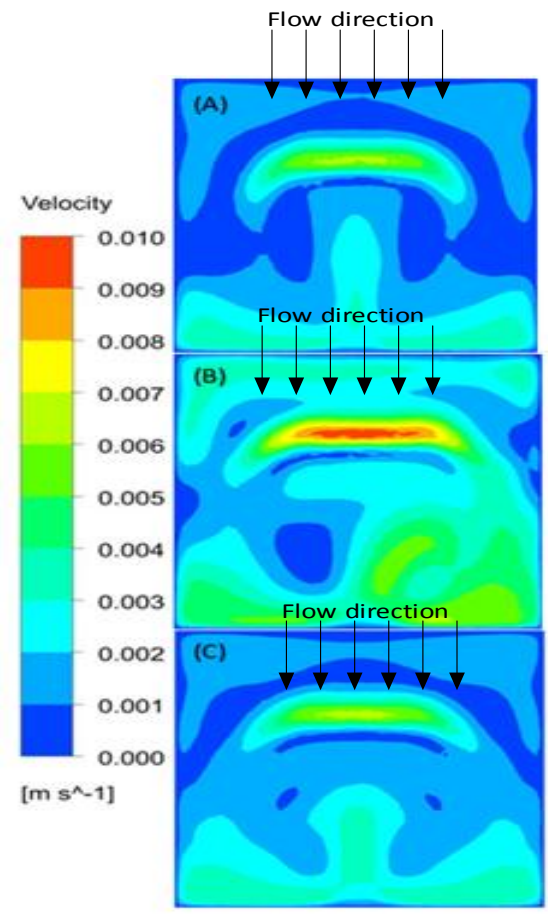

Fig 5: Velocity contour at plane $x-y(z=2.083 \mathrm{~m})$

\subsection{Temperature Distribution}

Fig. 6 shows the temperature distribution on the top surface of the transformer for all cases (note the air flow direction). In Case A, high temperature hot spot was observed at the middle of the surface. The maximum temperature at top of transformer is predicted at $345.1 \mathrm{~K}$. The upper part shows the cooler region compared to lower part the top of the transformer. This is because the air that flows from the inlet hits the transformer side panel and causes high heat being picked-up, leaving lower temperature region. The outer temperature surface shows lower temperature in the range of $338 \mathrm{~K}-342 \mathrm{~K}$. On-site measurement of transformer surface temperature was made to validate the simulation. Note that the measured surface temperature of the transformer ranges from 339-344K thus it can be said that comparison between the predicted and measure temperature show good agreement. This are later averaged for comparison with actual surface temperature. The comparison between measured and prediction is summarized in Table 3.

Table 3: Comparison of surface and air temperature at top transformer surface

\begin{tabular}{|l|c|c|}
\hline & Measured & Prediction \\
\hline Top transformer, ${ }^{\circ} \mathbf{C}$ & 68.7 & 69.5 \\
\hline
\end{tabular}

In Case B the temperature of air that flows out is predicted to be $310 \mathrm{~K}$ and $309 \mathrm{~K}$ at Outlet 1 and 2 respectively. Difference in temperature at Outlet 1 and 2 is due to the fact that the distance the air needs to travel to exit at Outlet 1 is longer than Outlet 2. Thus more heat is absorbed by flow path that exits via Outlet 1 as compared to Outlet 2. In terms of top transformer surface temperature, Case B shows lower transformer surface temperature as compared to Case A. The highest surface temperature of the transformer was predicted at a small region on right side where the fin is located.

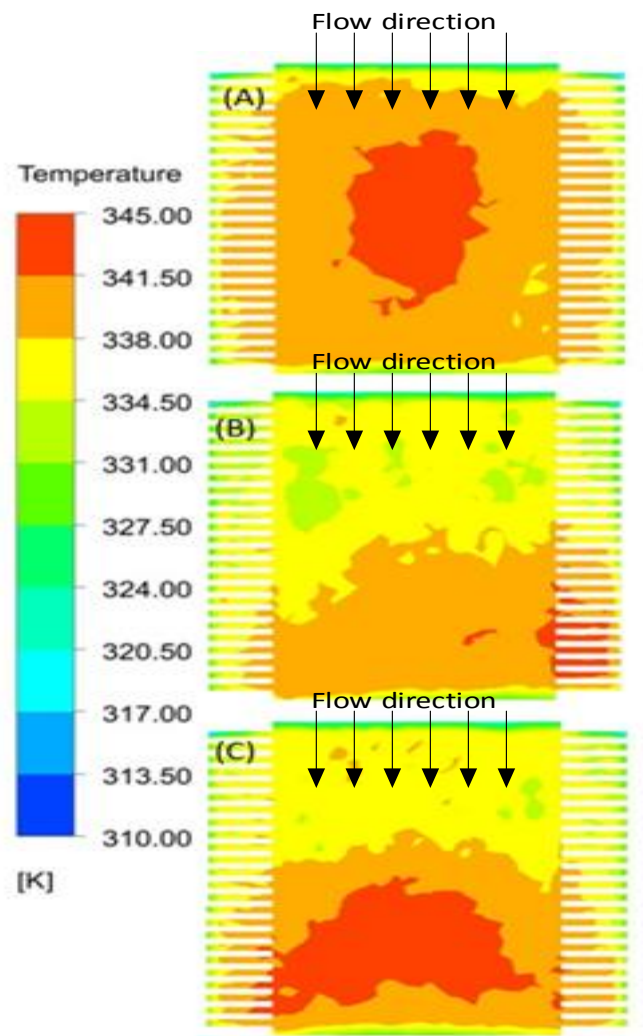

Fig 6: Temperature contour at transformer top for (A) Case A, (B) Case B and (C) Case C

Most of the transformer heat has been successfully convected, leaving lower surface temperature. The temperature at upstream surface is approximately $334 \mathrm{~K}$ while the downstream region has 
slightly higher surface temperature of $342 \mathrm{~K}$. In Case C, the average top transformer surface temperature is higher than the one given by Case B. Higher hot spot surface temperature of the transformer is predicted at transformer downstream with a magnitude of approximately $345 \mathrm{~K}$. Thus, by comparison of transformer surface temperature, it can be said that Case B give the best solution to reduce the transformer surface temperature.

To illustrate the temperature contours at mid plane of the substation, Fig. 7 shows the temperature contour along $y$ - $z$ plane. Generally, all cases show almost similar profiles except for different magnitude at critical regions. Air enters the building (from the left) at $300 \mathrm{~K}$ and as it passes through the transformer, heat is picked up and the surrounding temperature starts to increase. It is also noted that maximum temperature is given by temperature core with a magnitude of $355 \mathrm{~K}$. As heat is conducted towards the surface, the temperature reduces further and reaches $336 \mathrm{~K}$ at the surface.

Fig 8 shows the temperature contours at $y-z$ plane, parallel to the side surface of the transformer. Air enters the building from the left at $300 \mathrm{~K}$ and develops into all corners, picking up heat towards the outlets. All cases show reasonably similar trend except for the magnitude of surrounding air in particular at some regions To quantitatively assess the substation temperature from inlet to the outlet, a temperature profile is plotted at a fixed mid plane for consistency. This is shown in Fig. 9. The air temperature generally shows similar trend for all cases, except for the regions close to the top transformer surface. The highest temperature is given by Case A at $340 \mathrm{~K}$ and followed by Case C. The lowest air temperature is given by Case $\mathrm{B}$ and thus it can be said that the configuration given by Case $\mathrm{B}$ has the potential to give optimum heat dissipation performance for transformer cooling with minimum ventilation.

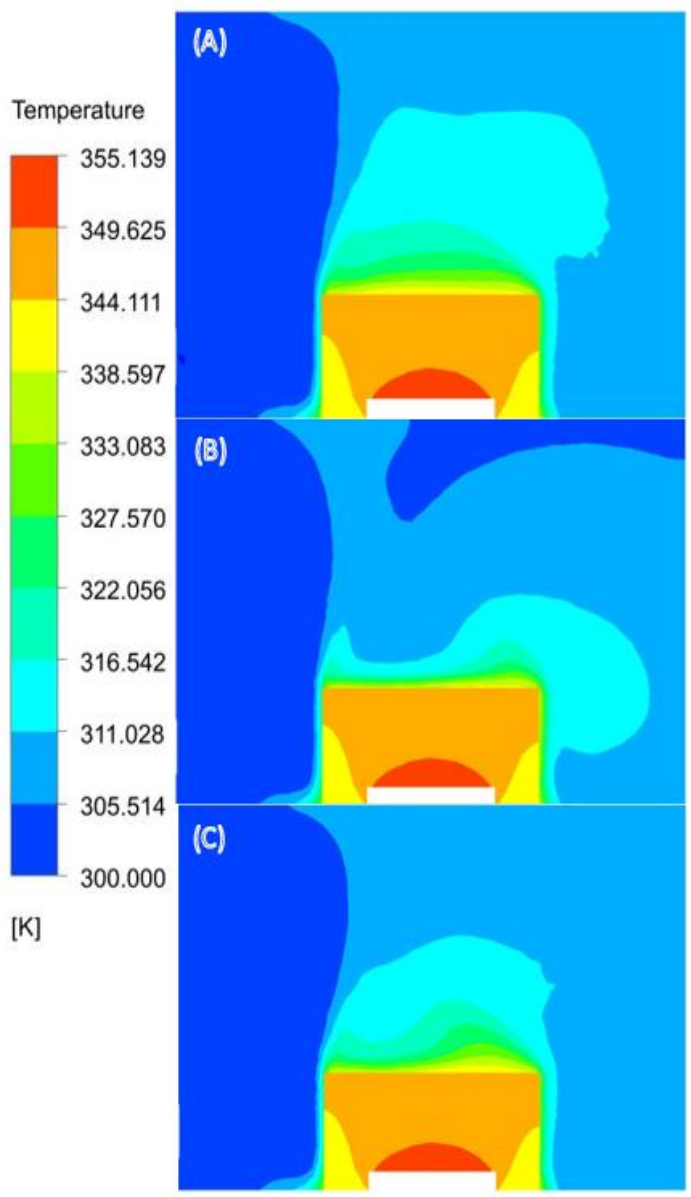

Fig 7: Temperature contour at $y-z$ plane $(x=2.98 \mathrm{~m})$

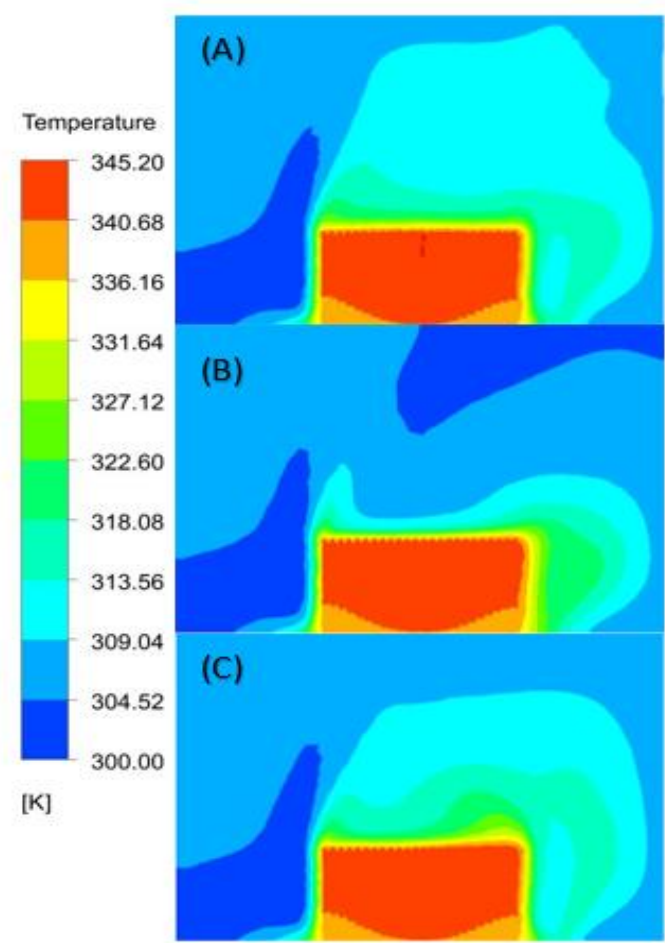

Fig 8: Temperature contour at plane $\mathrm{YZ}(\mathrm{x}=3.8545 \mathrm{~m})$

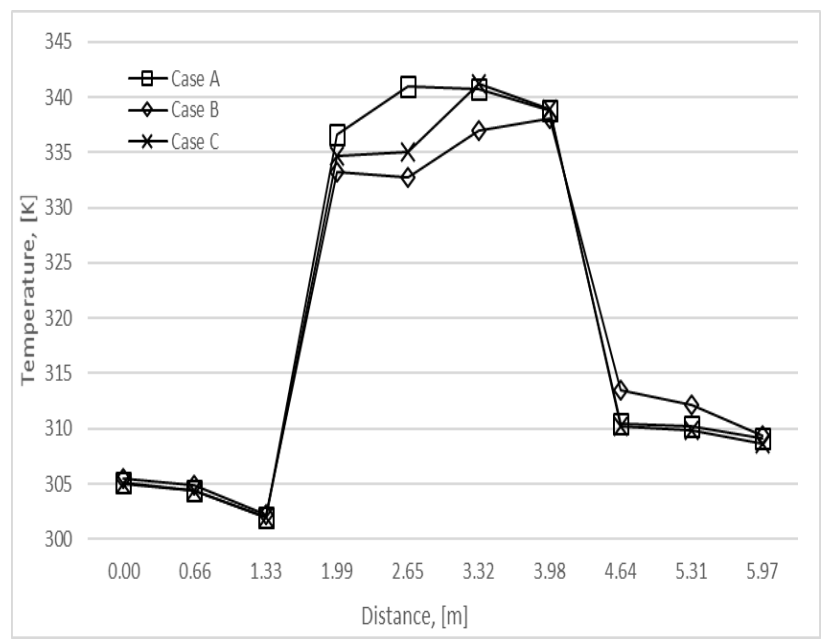

Fig 9: Profiles of local temperature against distance at centre plane

\section{Conclusion}

In this work, CFD simulation is carried out to investigate the performance of ventilation system for the transformer substation. Three different inlet and outlet configurations were tested and analysed based on the effectiveness in terms of transformer surface temperature and surrounding air. The prediction of transformer surface temperature shows good agreement with measure data on similar transformer in operation. Based on the simulation result, it can be said that air flow is well-distributed throughout the building, indicating good distribution to enhance cooling, regardless of the location of the outlet vents. In terms of transformer top surface prediction, Case B is able to give slightly better ventilation as it shows the lowest surface temperature compared to the other two cases.

\section{Acknowledgement}

The authors would also like to acknowledge the financial assistance received from UNITEN Internal Research Grant (UNIIG) that enable the work to be carried out. 


\section{References}

[1] Liu, H., Hao, Y., Fu, M., Wang, D., \& Yang, L. (2017). Study on ventilation of indoor substation main transformer room based on COMSOL software. 2017 1st International Conference on Electrical Materials and Power Equipment (ICEMPE). doi:10.1109/icempe.2017.7982088.

[2] Yu, T., Yang, H., Xu, R., \& Peng, C. (2014). Simulation Study on Ventilation \& Cooling for Main Transformer Room of an Indoor Substation.[Online]. Journal of Multimedia, 9(8). doi:10.4304/jmm.9.8.1040-1047.

[3] Smolka, J., \& Nowak, A. J. (2008). Experimental validation of the coupled fluid flow, heat transfer and electromagnetic numerical model of the medium-power dry-type electrical transformer.[Online]. International Journal of Thermal Sciences, 47(10), 1393-1410. doi:10.1016/j.ijthermalsci.2007.11.004

[4] Huijuan, Y., Tingfang, Y., Rui, X., \& Chunhua, P. (2015). Numerical Simulation of Ventilation for Main Transformer Room of Indoor Substations.[Online]. The Open Automation and Control Systems Journal, 7(1), 630-639. doi:10.2174/1874444301507010630

[5] Ramponi, R. and Blocken, B., "CFD simulation of cross-ventilation flow for different isolated building configurations: Validation with wind tunnel measurements and analysis of physical and numerical diffusion effects," Journal of Wind Engineering and Industrial Aerodynamics, vol. 104-106, pp. 408-418, 2012. 